\title{
Tunable Dirac cone in the topological insulator $\mathrm{Bi}_{2-x} \mathrm{Sb}_{\mathrm{x}} \mathrm{Te}_{3-\mathrm{y}} \mathrm{Se}_{\mathrm{y}}$
}

\author{
T. Arakane1, T. Sato ${ }^{2}$, S. Souma ${ }^{1}$, K. Kosaka², K. Nakayama², M. Komatsu², T. Takahashi¹,2, Zhi Ren ${ }^{3}$, \\ Kouji Segawa 3 \& Yoichi Ando 3
}

The three-dimensional topological insulator is a quantum state of matter characterized by an insulating bulk state and gapless Dirac cone surface states. Device applications of topological insulators require a highly insulating bulk and tunable Dirac carriers, which has so far been difficult to achieve. Here we demonstrate that $\mathrm{Bi}_{2-x} \mathrm{Sb}_{x} \mathrm{Te}_{3-y} \mathrm{Se}_{y}$ is a system that simultaneously satisfies both of these requirements. For a series of compositions presenting bulk-insulating transport behaviour, angle-resolved photoemission spectroscopy reveals that the chemical potential is always located in the bulk band gap, whereas the Dirac cone dispersion changes systematically so that the Dirac point moves up in energy with increasing $x$, leading to a sign change of the Dirac carriers at $x \sim 0.9$. Such a tunable Dirac cone opens a promising pathway to the development of novel devices based on topological insulators.

\footnotetext{
${ }^{1}$ WPI Research Center, Advanced Institute for Materials Research, Tohoku University, Sendai 980-8577, Japan. ${ }^{2}$ Department of Physics, Tohoku University, Sendai 980-8578, Japan. ${ }^{3}$ Institute of Scientific and Industrial Research, Osaka University, Ibaraki, Osaka 567-0047, Japan. Correspondence and requests for materials should be addressed to T.S. (email: t-sato@arpes.phys.tohoku.ac.jp).
} 
T he surface state of a three-dimensional topological insulator (TI) is characterized by a Dirac cone dispersion showing a helical spin structure ${ }^{1-4}$, which makes the Dirac fermions immune to backward scattering and robust to non-magnetic impurities and disorder ${ }^{5,6}$. Experimental realizations of novel topological phenomena expected for such helical Dirac fermions hinge on the dominance of surface transport, but a highly insulating bulk has rarely been achieved in prototypical TIs such as $\mathrm{Bi}_{2} \mathrm{Se}_{3}$ and $\mathrm{Bi}_{2} \mathrm{Te}_{3}$ because of naturally occurring defects and the resulting carrier doping ${ }^{3,7-10}$. Furthermore, applications of TIs to a wide range of devices would require a means to intentionally manipulate the properties of the Dirac carriers (sign, density, velocity and so on.) while keeping the bulk sufficiently insulating. However, such a Dirac cone engineering has been difficult to achieve because of the lack of suitable materials.

Recently, it has been shown that the ternary tetradymite TI material $\mathrm{Bi}_{2} \mathrm{Te}_{2} \mathrm{Se}$, which forms the ordered $\mathrm{Te}-\mathrm{Bi}-\mathrm{Se}-\mathrm{Bi}-\mathrm{Te}$ quintuple layers, has a large bulk resistivity ${ }^{11,12}$ because of its chemical characteristics suitable for reducing defect formations. In this regard, the tetradymite $\mathrm{Bi}_{2-x} \mathrm{Sb}_{x} \mathrm{Te}_{3-y} \mathrm{Se}_{y}$ (BSTS) solid solution, which has the same crystal structure as $\mathrm{Bi}_{2} \mathrm{Te}_{2} \mathrm{Se}$ (Fig. 1a), is of interest because a series of special combinations of $x$ and $y$ have been known to yield a high resistivity ${ }^{13-15}$. Thus, in the insulating compositions reported here $((x, y)=(0,1),(0.25,1.1),(0.5,1.3),(1,2))$, the $y$ value is unique when $x$ is specified (details are described in Methods (in the subsection Transport properties of BSTS)). Such a control of the material properties is an advantage of the solid-solution systems ${ }^{16-18}$ and makes the BSTS system an interesting platform for investigating the Dirac band structure while keeping the insulating nature of the bulk.

Here we report our angle-resolved photoemission spectroscopy (ARPES) experiments on BSTS, which elucidated the surface and bulk electronic states in the vicinity of the Fermi level $\left(E_{\mathrm{F}}\right)$ responsible for the peculiar physical properties. We show that simultaneous tuning of the Sb and Se contents in the BSTS crystal makes it possible to control the energy location of the Dirac cone in the bulk band gap (and the sign of Dirac carriers) while keeping the bulk insulating character. This result demonstrates that what BSTS offers is, at present, the closest to the ultimate goal of the Dirac cone engineering, that is, being able to tune the Dirac band structure to have desired surface carrier properties without having to tune the chemical potential in bulk crystals.

\section{Results}

Electronic states of $\mathbf{B i}_{2} \mathrm{Te}_{2} \mathrm{Se}$. We first demonstrate the ARPES data of an end member $\mathrm{Bi}_{2} \mathrm{Te}_{2} \mathrm{Se}(x=0 ; y=1)$. The photon-energy dependence of the band structure near $E_{\mathrm{F}}$ is displayed in Figure $1 \mathrm{~b}$, where one can see several common features such as a prominent electron-like band centred at the $\bar{\Gamma}$ point $\left(k_{\mathrm{y}}=0\right)$ in the binding energy $E_{\mathrm{B}}$ range of $0.0-0.3 \mathrm{eV}$ and rather complicated band dispersions at $E_{\mathrm{B}}>0.3 \mathrm{eV}$. These features correspond to the surface state (SS) and the bulk valence bands (VB), respectively, judged from the analysis shown in Figure 1c, where the energy position of the SS is stationary with the variation in $h v$ unlike that of the VB. A closer look at Figure 1c also reveals that the SS has an ' $\mathrm{x}$ '-shaped dispersion with its Dirac point at $\sim 0.3 \mathrm{eV}$, indicative of an electrondoped character of the surface. The highest lying VB at $\sim 0.4 \mathrm{eV}$ exhibits an ' $m$ '-shaped dispersion and is located closest to $E_{\mathrm{F}}$ at $h v=58 \mathrm{eV}$. We use this photon energy for comparing the electronic states at different compositions of BSTS and also for quantitatively estimating characteristic energies, as will be described later. One can see in Figure $1 \mathrm{~b}$ that the signature of the bulk conduction band (CB) is completely absent in the ARPES intensity, confirming the insulating nature of the bulk. The two-dimensional contour plots of the ARPES intensity at various $E_{\mathrm{B}}$ shown in Figure $1 \mathrm{~d}$ signify the hexagonal warping of the SS band structure that gradually weakens on approaching the Dirac point, as commonly observed in other TIs $^{4,8,19}$. Outside the SS, the VB feature is visible as a six-fold petallike intensity pattern at $E_{\mathrm{B}}=0.25-0.3 \mathrm{eV}$.
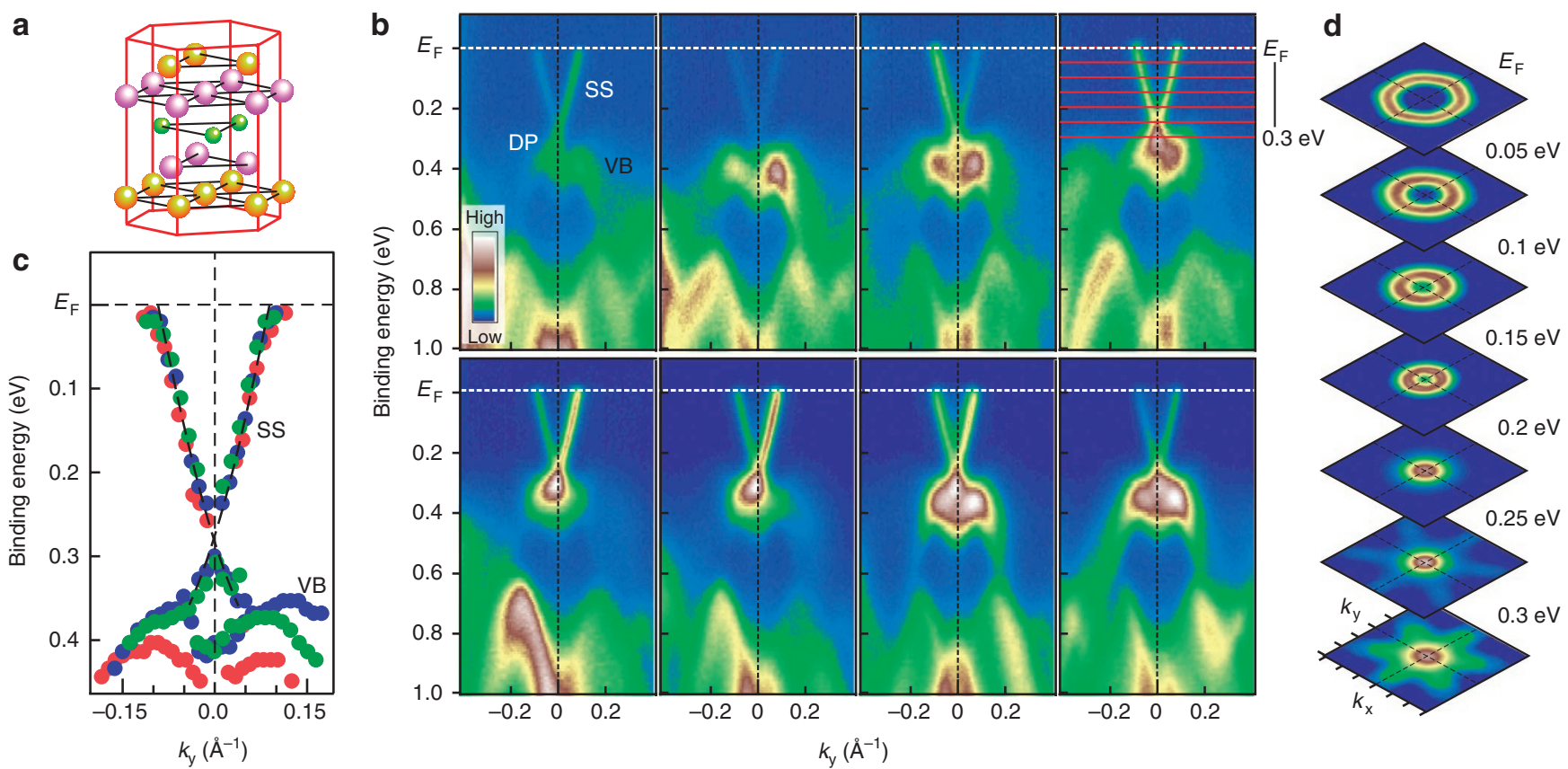

Figure 1 | Photon energy dependence of the band structure in $\mathbf{B i}_{\mathbf{2}} \mathbf{T e}_{\mathbf{2}} \mathbf{S e}$. (a) Crystal structure of $\mathrm{Bi}_{2-x} \mathrm{Sb}_{x} T e_{3-y} \mathrm{Se}_{y}$. Green, purple and yellow circles represent $\mathrm{Se}, \mathrm{Bi} / \mathrm{Sb}$ and Te/Se atoms, respectively. (b) Photon energy dependence of the ARPES intensity around the $\bar{\Gamma}$ point for $\mathrm{Bi}_{2} \mathrm{Te} \mathrm{S}_{2} \mathrm{Se}(x=0, y=1)$ measured at $h v=46,50,54$ and $58 \mathrm{eV}$ (top row) and 62,66,70 and $74 \mathrm{eV}$ (bottom row) at $T=30 \mathrm{~K}$. (c) Comparison of the band dispersions of the SS and the bulk VB in $x=0$ for 3 representative photon energies $h v=50,58$ and $70 \mathrm{eV}$, as indicated by red, blue and green circles, respectively. The band dispersions were obtained by tracing the peak position of EDCs. Black dashed curves are guides to the eyes to trace the SS. (d) ARPES intensity plots for $x=0$ as a function of two-dimensional wave vector measured with $h v=58 \mathrm{eV}$, for various binding energies between $E_{\mathrm{F}}$ and $0.3 \mathrm{eV}$. The intensity maps were obtained by folding the ARPES intensity with the crystal symmetry taken into account. 
Evolution of electronic states in $\mathrm{Bi}_{2-\mathrm{x}} \mathrm{Sb}_{\mathrm{x}} \mathrm{Te}_{3-\mathrm{y}} \mathrm{Se}_{\mathrm{y}}$. Figure $2 \mathrm{a}-\mathrm{c}$ show a comparison of the Fermi surface and the near- $E_{\mathrm{F}}$ band structure at different compositions, all of which belong to the bulk insulating phase as confirmed by the resistivity data shown in Figure 2d. Indeed, only the $\mathrm{SS}$ is present at $E_{\mathrm{F}}$ in all the samples. Interestingly, the SS Fermi surface systematically shrinks on increasing $x$ (Fig. 2a) accompanied by an overall upward shift of the SS (Fig. 2b,c). This demonstrates that increasing $x$ (and the simultaneous increase in $y$ ) provides more acceptors, which is likely due to a slight change in the carrier compensation condition, while the bulk remains highly insulating as confirmed by the electrical resistivity data in Figure 2d; in particular, the resistivity for $\mathrm{Bi}_{1.5} \mathrm{Sb}_{0.5} \mathrm{Te}_{1.7} \mathrm{Se}_{1.3}(x=0.5 ; y=1.3)$ is very high for TIs, reaching $10 \Omega \mathrm{cm}$ at low temperatures, and previous magnetotransport studies elucidated the surface mobility to exceed $1,000 \mathrm{~cm}^{2} \mathrm{Vs}^{-1}$ for this composition ${ }^{14}$. An important indication of our data is a systematic compensation of Dirac carriers that can be seen in Figure 2a-c; this trend can also be confirmed in the momentum distribution curves (MDCs) at $E_{\mathrm{F}}$ shown in Figure 2e, where the momentum separation of two peaks in the MDC, corresponding to the $2 k_{\mathrm{F}}$ (Fermi vector) value, gradually decreases with increasing $x$. To quantitatively evaluate the evolution of the SS, we plot in Figure $2 \mathrm{f}$ the Dirac band dispersion determined from the peak positions of the energy distribution curves (EDCs) in Figure 2c. One can see that the energy shift of the Dirac band proceeds in a rigid-band manner, the bands for different $x$ values essentially overlap with each other when we plot its energy position with respect to the Dirac-point energy $\left(E_{\mathrm{DP}}\right)$, despite the total chemical potential $(\mu)$ shift of as large as $0.3 \mathrm{eV}$ as seen in Figure $2 \mathrm{~g}$. Intriguingly, the $\mathrm{SS}$ band dispersion below $E_{\mathrm{F}}$ for $x=1.0$ suggests that the Dirac point is located slightly above $E_{\mathrm{F}}$, which can also be confirmed in the plot of the $2 k_{\mathrm{F}}$ values in Figure $2 \mathrm{~h}$, pointing to a sign change of Dirac carriers from $n$ - to $p$-type at some $x$ value between 0.5 and 1.0. Another important indication in Figure 2 is that the bulk VB does not show a rigid-band shift relative to the SS, as inferred from the data in Figure 2b, where the lower hole-like branch of the Dirac cone for $x=1.0$ is more clearly visible than in $x=0$.

Band diagram of $\mathrm{Bi}_{2-\mathrm{x}} \mathrm{Sb}_{\mathrm{x}} \mathrm{Te}_{3-\mathrm{y}} \mathrm{Se}_{\mathrm{y}}$. Looking at Figure 2a, one notices that the Dirac point is buried in the bulk VB at $x=0$, which means that the putative transport properties near the Dirac point would be strongly affected by the bulk-surface interband scattering. In contrast, one can see in Figure $2 \mathrm{~b}$ that the Dirac point for $x=1.0$ is well isolated from the bulk, indicating a more ideal situation for applications. To depict a comprehensive picture of the evolution of the energy bands, it is necessary to determine the energy locations of the bulk-band edges. This requires the observation of the $\mathrm{CB}$ which becomes possible by ageing the sample surface ${ }^{20-22}$. The details of this ageing experiments are described in Methods (Surface ageing to observe the bulk $\mathrm{CB}$ ). We have estimated $E_{\mathrm{CB}}$ and $E_{\mathrm{VB}}$ as well as the Dirac-point energy $\left(E_{\mathrm{DP}}\right)$ by using the ageing technique for all compositions, and the obtained characteristic energies are shown in Figure $3 a$. While the magnitude of the band gap is almost independent of $x$, the Dirac-point energy relative to the VB top, $E_{\mathrm{DP}}-E_{\mathrm{VB}}$, is negative at $x=0$ and turns to positive around the critical $x$ of $\sim 0.25$. As illustrated in the schematic band diagram (Fig. 3b), the intrinsic transport properties near the Dirac point can be achieved in samples with $x>0.25$, and, therefore, this composition range is particularly suited for realizing the topological phenomena to require the tuning of $\mu$ to the Dirac point, such as the topological magnetoelectric effect $^{23}$. Another important aspect is that $\mu$ at the native surface of our BSTS samples is located above the Dirac point in $0 \leq x \leq 0.5$ and below it in $x=1.0$. This suggests that a sign change in surface Dirac carriers takes place at $x \sim 0.9$ (estimated from a linear interpolation in Fig. $2 \mathrm{~h}$ ), and, therefore, a $p-n$ junction fabricated by a composition gradient in BSTS may be conceivable. In this regard, the Hall coefficient at low temperature is negative for $x=0.0-0.5$, whereas it is positive for $x=1.0$ as described in Methods (in the subsection Transport properties of BSTS), in good agreement with the ARPES data.

\section{Discussion}

The observed isolated nature of the Dirac cone at $x=1$ as opposed to its buried character at $x=0$ may be understood in terms of the difference in the Se/Te content: according to the previous ARPES studies, the Dirac point in $\mathrm{Bi}_{2} \mathrm{Se}_{3}$ (ref. 3) is well isolated from the bulk bands while that in $\mathrm{Bi}_{2} \mathrm{Te}_{3}$ is situated inside the bulk $\mathrm{VB}^{8}$. In BSTS, the Dirac cone is gradually isolated from the bulk band as the $\mathrm{Se} / \mathrm{Te}$ ratio is increased from 0.5 to 2.0 (on which $x$ changes from 0.0 to 1.0), in accordance with the natural expectation from the difference in the electronic states in $\mathrm{Bi}_{2} \mathrm{Se}_{3}$ and $\mathrm{Bi}_{2} \mathrm{Te}_{3}$. This suggests the importance of controlling the orbital character of chalcogenderived bands for the Dirac cone engineering.

The experimental realization of both Dirac holes and electrons in the BSTS system demonstrated here points to the high potential of this material for studying the various topological phenomena requiring the access to the Dirac point. Moreover, it would provide an excellent platform for the development of novel topological devices to utilize a dual-gate configuration for the electric control of spins $^{24}$ or $p-n$-junction configurations that are essential for various applications, as in semiconductor technology. Another important feature of this system is that the energy location of the Dirac point in the band gap can be tuned while keeping the bulk-insulating nature and a high surface mobility, allowing one to study the effect of the bulk-surface scattering channel on the surface carriers near the Dirac point. Furthermore, the availability of the Dirac cone engineering in bulk crystals is important for the topological magnetoelectric effect, because it has been proposed that the energy gain in the bulk owing to the axion term can be crucial for realizing such an effect ${ }^{25}$. The present result provides an important step toward establishing the means to fully control of surface Dirac fermions in TIs to explore a variety of exotic physical properties proposed for this exciting class of materials.

\section{Methods}

Sample preparation. High-quality single crystals of BSTS were grown by sealing stoichiometric amounts of high-purity elements in evacuated quartz tubes and melting them at $850^{\circ} \mathrm{C}$ for $48 \mathrm{~h}$ with intermittent shaking to ensure a homogeneity of the melt, followed by cooling slowly to $550^{\circ} \mathrm{C}$ and annealing at that temperature for 4 days. X-ray diffraction analyses confirmed that all the samples have the same crystal structure $(R \overline{3} \mathrm{~m})$ with the desired chalcogen ordering as shown in Figure la. Transport properties were measured with Quantum Design PPMS using the standard AC four-probe method.

ARPES experiments. ARPES measurements were performed with a VG Scienta SES2002 electron analyser with a tunable synchrotron light at the beamline BL28A at Photon Factory (KEK). We used circularly polarized lights of $36-116 \mathrm{eV}$. The energy and angular resolutions were set at $15-30 \mathrm{meV}$ and $0.2^{\circ}$, respectively. ARPES measurements were also performed with a MBS A1 electron analyzer with a highflux Xe discharge lamp and a spherical grating monochromator $(h v=8.437 \mathrm{eV})^{26}$ at Tohoku University. Samples were cleaved in-situ along the (111) crystal plane in an ultrahigh vacuum of $1 \times 10^{-10}$ Torr. The Fermi level of the samples was referenced to that of a gold film evaporated onto the sample holder. A shiny mirror-like surface was obtained after cleaving the samples, confirming its high quality.

Transport properties of BSTS. As was reported in ref. 15, the 'intrinsic' compositions in the solid-solution system BSTS were recently elucidated. At such compositions, acceptors and donors maximally compensate each other, and bulkinsulating behaviour is observed. In the present ARPES experiment, we studied four combinations of $(x, y)[=(0,1),(0.25,1.1),(0.5,1.3)$, and $(1,2)]$ that all belong to such intrinsic compositions. The crystals used in the present work are obtained after careful optimization of the growth conditions for insulating behaviour, as evidenced by the resistivity data (Fig. 2 d) that present even higher values for all compositions compared with those reported in ref. 15 .

To corroborate our claim that all the compositions studied here possess highly bulk-insulating nature, and to elucidate the sign of the surface charge carriers, the temperature dependences of the Hall coefficient $R_{\mathrm{H}}$ for the four compositions were measured (Supplementary Fig. S1). The Hall measurements were done on the same samples as those used for the resistivity measurements. One can see that the 
a

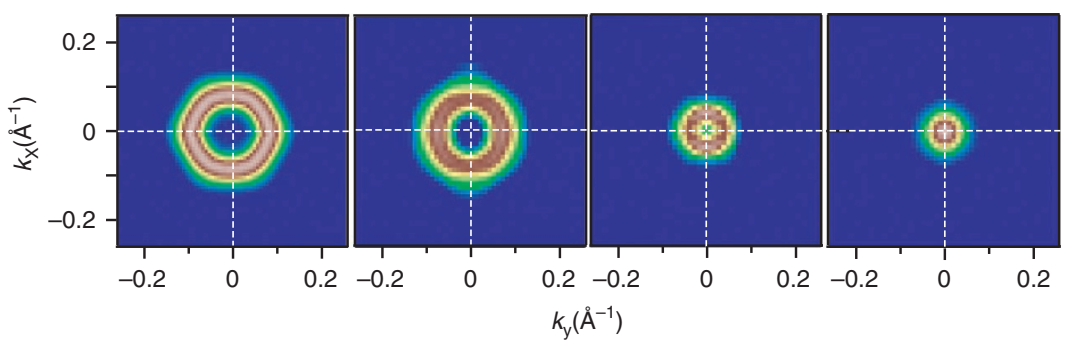

b

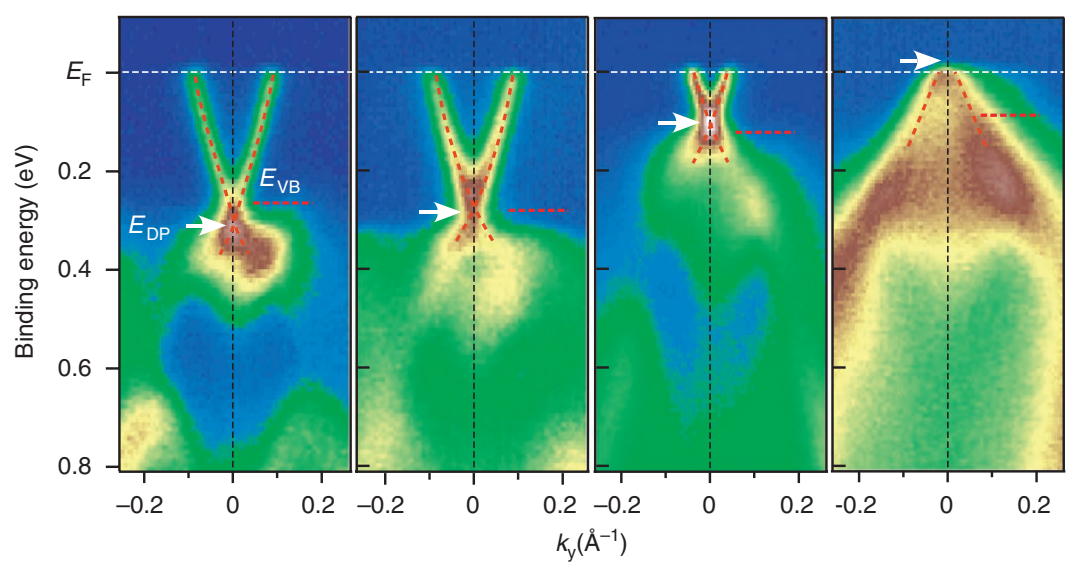

C


e
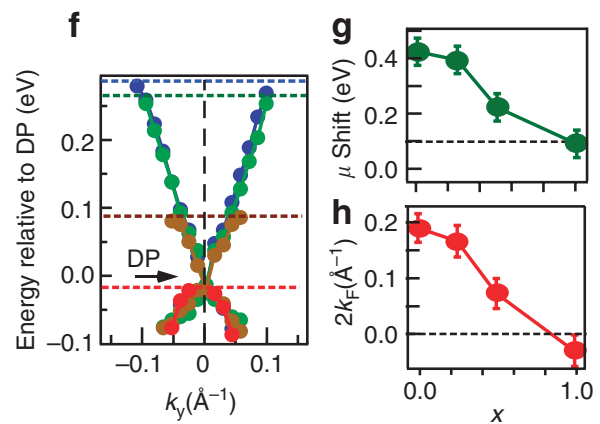

Figure $\mathbf{2}$ | Properties of $\mathbf{B i}_{\mathbf{2}-\mathbf{x}} \mathbf{S b}_{\mathbf{x}} \mathbf{T e}_{\mathbf{3}-\mathbf{y}} \mathbf{S} \mathbf{e}_{\mathbf{y}}$ for various compositions. (a-c) Comparison of (a) the Fermi surface of the SS, (b) the near- $E_{\mathrm{F}}$ band dispersions, and (c) the EDCs in $\mathrm{Bi}_{2-x} \mathrm{Sb}_{x} T e_{3-y} \mathrm{Se}_{y}$ for four $x$ values $(x=0,0.25,0.5$ and 1.0$)$ where $y=1,1.15,1.3$ and 2 , respectively. The ARPES data were measured with $h v=58 \mathrm{eV}$ at $T=30 \mathrm{~K}$. Brown dashed curves in (b) are guides to the eyes to trace the SS. White arrows and red dashed lines indicate the energy positions of the Dirac point $\left(E_{\mathrm{DP}}\right)$ and the $\mathrm{VB}$ top $\left(E_{\mathrm{VB}}\right)$, respectively. (d) Temperature dependences of the electrical resistivity $\rho_{x x}$ at the four compositions. (e-h) Composition dependences of (e) the MDCs at $E_{F}(\mathbf{f})$ the band dispersions of the SS relative to the Dirac point, $(\mathbf{g})$ the shift of the chemical potential $(\mu)$ relative to that for $x=1.0$ when the Dirac point is taken as the reference point, and (h) the $2 k_{\mathrm{F}}$ values along $k_{\mathrm{y}} \cdot k_{\mathrm{F}}$ values were determined by fitting MDCs at $E_{\mathrm{F}}$ with two Lorentzian peaks. Blue, green, brown and red curves or dots in $\mathbf{d}-\mathbf{f}$ are for $x=0,0.25,0.5$ and 1.0 , respectively. Error bars in ( $\mathbf{g}$ ) correspond to the experimental uncertainty in determining $E_{\mathrm{DP}}$, whereas those in $(\mathbf{h})$ originates in the experimental uncertainty in estimating the peak position of MDCs. 


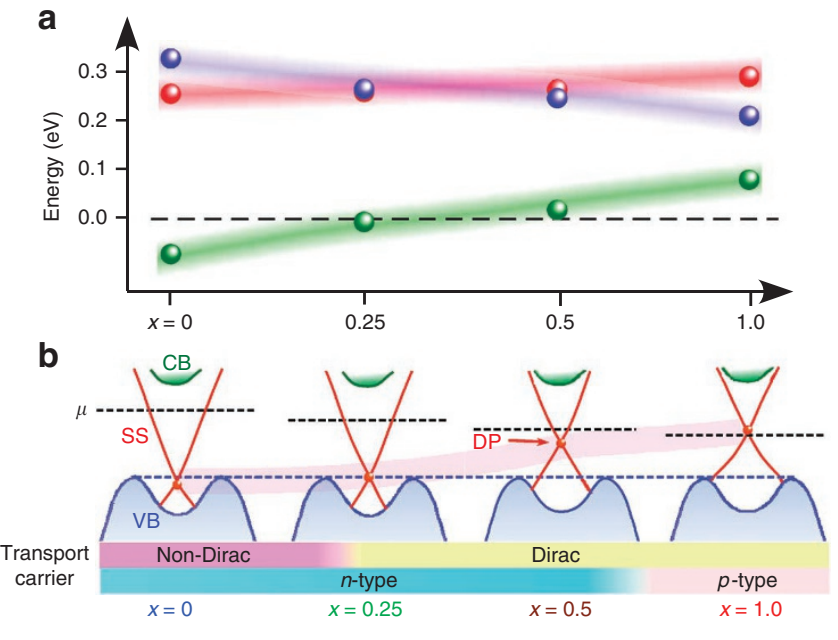

Figure 3 | Characteristic energies and the schematic band diagram.

(a) Plot of the three characteristic energies, the band gap $E_{\text {gap }}\left(=E_{\mathrm{CB}}-E_{\mathrm{VB}}\right)$, $E_{C B}-E_{D P}$, and $E_{D P}-E_{V B}$, as a function of $x$, as indicated by red, blue and green colours, respectively. (b) Schematic band diagram for changing $x$ in BSTS derived from the present ARPES experiment. The top of the VB is aligned in this diagram for clarity.

absolute values of $R_{\mathrm{H}}$ at the lowest temperature are $400 \mathrm{~cm}^{3}$ per ${ }^{\circ} \mathrm{C}$ or larger, which indicates that the present samples are even better insulators than the $\mathrm{Bi}_{2} \mathrm{Te}_{2} \mathrm{Se}$ sample originally reported in ref. 13 (where $\left|R_{\mathrm{H}}\right|$ at the lowest temperature was $200 \mathrm{~cm}^{3}$ per $\left.{ }^{\circ} \mathrm{C}\right)$.

Surface ageing to observe the bulk conduction band. To determine the energy location of the bulk $\mathrm{CB}$ that is located above $E_{\mathrm{F}}$ in our insulating samples, we intentionally aged the sample surface in our vacuum chamber and doped electrons into the SS to enhance the surface band-bending effect, by following the ARPES studies of $\mathrm{Bi}_{2} \mathrm{Se}_{3}$ and $\mathrm{Bi}_{2} \mathrm{Te}_{3}$ (refs 20-22) and also a recent transport study of BSTS $^{14}$. A representative result for $x=0$ shown in Supplementary Figure S2 demonstrates that the ageing leads to an overall downward shift of the spectral feature and, simultaneously, the appearance of the $\mathrm{CB}$ at $E_{\mathrm{F}}$. As shown in the right panel of Supplementary Figure S2, it is thus possible to estimate the actual locations of the bottom of the $\mathrm{CB}\left(E_{\mathrm{CB}}\right)$ and the top of the $\mathrm{VB}\left(E_{\mathrm{VB}}\right)$ by tracing the trailing/leading edges of the EDCs ${ }^{27}$.

\section{References}

1. Hasan, M. Z. \& Kane, C. L. Colloquium: topological insulators. Rev. Mod. Phys. 82, 3045-3067 (2010).

2. Qi, X.- L. \& Zhang, S.- C. Topological insulators and superconductors. Rev. Mod. Phys. 83, 1057-1110 (2011).

3. Xia, Y. et al. Observation of a large-gap topological-insulator class with a single Dirac cone on the surface. Nature Phys. 5, 398-402 (2009).

4. Souma, S. et al. Direct measurement of the out-of-plane spin texture in the Dirac-cone surface state of a topological insulator. Phys. Rev. Lett. 106, 216803 (2011).

5. Roushan, P. et al. Topological surface states protected from backscattering by chiral spin texture. Nature 460, 1106-1109 (2009).

6. Seo, J. et al. Transmission of topological surface states through surface barriers. Nature 466, 343-346 (2010).

7. Hsieh, D. et al. A topological Dirac insulator in a quantum spin Hall phase. Nature 452, 970-974 (2008).

8. Chen, Y.- L. et al. Massive Dirac fermion on the surface of a magnetically doped topological insulator. Science 329, 659-662 (2010).

9. Hor, Y. S. et al. p-type $\mathrm{Bi}_{2} \mathrm{Se}_{3}$ for topological insulator and low-temperature thermoelectric applications. Phys. Rev. B 79, 195208 (2009).
10. Checkelsky, J. G. et al. Quantum interference in macroscopic crystals of nonmetallic $\mathrm{Bi}_{2} \mathrm{Se}_{3}$. Phys. Rev. Lett. 103, 246601 (2009).

11. Ren, Z., Taskin, A. A., Sasaki, S., Segawa, K. \& Ando, Y. Large bulk resistivity and surface quantum oscillations in the topological insulator $\mathrm{Bi}_{2} \mathrm{Te}_{2} \mathrm{Se}$. Phys. Rev. B 82, 241306 (2010).

12. Xiong, J., Petersen, A. C., Qu, D., Cava, R. J. \& Ong, N. P. Quantum oscillations in a topological insulator $\mathrm{Bi}_{2} \mathrm{Te}_{2} \mathrm{Se}$ with large bulk resistivity $(6 \Omega \mathrm{cm})$. Preprint arXiv:1101.1315 (2011).

13. Teramoto, I. \& Takayanagi, S. Relations between the electronic properties and the chemical bonding of $\mathrm{Sb}_{\mathrm{x}} \mathrm{Bi}_{2-\mathrm{x}} \mathrm{Te}_{3-\mathrm{y}} \mathrm{Se}_{\mathrm{y}}$ system. J. Phys. Chem. Solids 19, 124-129 (1961).

14. Taskin, A. A., Ren, Z., Sasaki, S., Segawa, K. \& Ando, Y. Observation of Dirac holes and electrons in a topological insulator. Phys. Rev. Lett. 107, 016801 (2011).

15. Ren, Z., Taskin, A. A., Sasaki, S., Segawa, K. \& Ando, Y. Optimizing $\mathrm{Bi}_{2-\mathrm{x}} \mathrm{Sb}_{\mathrm{x}} \mathrm{Te}_{3-\mathrm{y}} \mathrm{Se}_{\mathrm{y}}$ solid solutions to approach the intrinsic topological insulator regime. Phys. Rev. B 84, 165311 (2011).

16. Taskin, A. A. \& Ando, Y. Quantum oscillations in a topological insulator $B_{1}$. ${ }_{\mathrm{x}} \mathrm{Sb}_{\mathrm{x}}$. Phys. Rev. B 80, 085303 (2009).

17. Zhang, J. et al. Band structure engineering in $\left(\mathrm{Bi}_{1-\mathrm{x}} \mathrm{Sb}_{\mathrm{x}}\right)_{2} \mathrm{Te}_{3}$ ternary topological insulators. Nat. Commun. 2, 574 (2011)

18. Kong, D. et al. Ambipolar field effect in topological insulator nanoplates of $\left(\mathrm{Bi}_{\mathrm{x}} \mathrm{Sb}_{1-\mathrm{x}}\right)_{2} \mathrm{Te}_{3}$. Nat. Nanotechnol. 6, 705-709 (2011).

19. Kuroda, K. et al. Hexagonally deformed Fermi surface of the 3D topological insulator $\mathrm{Bi}_{2} \mathrm{Se}_{3}$. Phys. Rev. Lett. 105, 076802 (2010).

20. Bianchi, M. et al. Coexistence of the topological state and a two-dimensional electron gas on the surface of $\mathrm{Bi}_{2} \mathrm{Se}_{3}$. Nat. Commun. 1, 128 (2010).

21. Wray, L. A. et al. Electron dynamics in topological insulator semiconductormetal interfaces (topological p-n interface). Preprint arXiv:1105.4794 (2011).

22. Benia, H. M., Lin, C., Kern, L. K. \& Ast, C. R. Reactive chemical doping of the $\mathrm{Bi}_{2} \mathrm{Se}_{3}$ topological insulator. Phys. Rev. Lett. 107, 177602 (2011).

23. Qi, X.- L., Hughes, T. L. \& Zhang, S.- C. Topological field theory of timereversal invariant insulators. Phys. Rev. B 78, 195424 (2008).

24. Yazyev, O. V., Moore, J. E. \& Louie, S. G. Spin polarization and transport of surface states in the topological insulators $\mathrm{Bi}_{2} \mathrm{Se}_{3}$ and $\mathrm{Bi}_{2} \mathrm{Te}_{3}$ from first principles. Phys. Rev. Lett. 105, 266806 (2010).

25. Nomura, K. \& Nagaosa, N. Surface-quantized anomalous Hall current and the magnetoelectric effect in magnetically disordered topological insulators. Phys. Rev. Lett. 106, 166802 (2011).

26. Souma, S., Sato, T., Takahashi, T. \& Baltzer, P. High-intensity xenon plasma discharge lamp for bulk-sensitive high-resolution photoemission spectroscopy. Rev. Sci. Instrum. 78, 123104 (2007).

27. Sato, T. et al. Direct evidence for the Dirac-cone topological surface states in ternary chalcogenide $\mathrm{TlBiSe}_{2}$. Phys. Rev. Lett. 105, 136802 (2010).

\section{Acknowledgements}

We thank Y. Tanaka, K. Yoshimatsu, H. Kumigashira and K. Ono for their assistance in ARPES measurements. This work was supported by JSPS (NEXT Program and KAKENHI 23224010), JST-CREST, MEXT of Japan (Innovative Area 'Topological Quantum Phenomena'), AFOSR (AOARD 10-4103), and KEK-PF (Proposal number: 2010G507).

\section{Author contributions}

T.A, T.S., S.S., K.K., K.N., M.K. and T.T. performed ARPES measurements. Z.R., K.S. and Y.A. carried out the growth of the single crystals and their characterizations. T.A., T.S., Z. R. and Y.A. conceived the experiments and wrote the manuscript.

\section{Additional information}

Supplementary Information accompanies this paper at http://www.nature.com/ naturecommunications

Competing financial interests: The authors declare no competing financial interests.

Reprints and permission information is available online at http://npg.nature.com/ reprintsandpermissions/

How to cite this article: Arakane, T. et al. Tunable Dirac cone in the topological insulator $\mathrm{Bi}_{2-\mathrm{x}} \mathrm{Sb}_{\mathrm{x}} \mathrm{Te}_{3-\mathrm{y}} \mathrm{Se}_{\mathrm{y}}$. Nat. Commun. 3:636 doi: 10.1038/ncomms1639 (2012). 